\title{
Metastable Atom-Activated Dissociation Mass Spectrometry of Phosphorylated and Sulfonated Peptides in Negative Ion Mode
}

\author{
Shannon L. Cook, Glen P. Jackson
}

Center for Intelligent Chemical Instrumentation, Department of Chemistry and Biochemistry, Ohio University, Athens, $\mathrm{OH}$, 45701-2979, USA

\begin{abstract}
The dissociation behavior of phosphorylated and sulfonated peptide anions was explored using metastable atom-activated dissociation mass spectrometry (MAD-MS) and collision-induced dissociation (CID). A beam of high kinetic energy helium $(\mathrm{He})$ metastable atoms was exposed to isolated phosphorylated and sulfonated peptides in the 3- and 2- charge states. Unlike CID, where phosphate losses are dominant, the major dissociation channels observed using MAD were $\mathrm{C}_{\alpha}-\mathrm{C}$ peptide backbone cleavages and neutral losses of $\mathrm{CO}_{2}, \mathrm{H}_{2} \mathrm{O}$, and $\left[\mathrm{CO}_{2}+\mathrm{H}_{2} \mathrm{O}\right]$ from the charge reduced (oxidized) product ion, consistent with an electron detachment dissociation (EDD) mechanism such as Penning ionization. Regardless of charge state or modification, MAD provides ample backbone cleavages with little modification loss, which allows for unambiguous PTM site determination. The relative abundance of certain fragment ions in MAD is also demonstrated to be somewhat sensitive to the number and location of deprotonation sites, with backbone cleavage somewhat favored adjacent to deprotonated sites like aspartic acid residues. MAD provides a complementary dissociation technique to CID, ECD, ETD, and EDD for peptide sequencing and modification identification. MAD offers the unique ability to analyze highly acidic peptides that contain few to no basic amino acids in either negative or positive ion mode.
\end{abstract}

Key words: Tandem mass spectrometry, MS/MS, Negative ion mode, Peptide fragmentation, Peptide anions, Post-translational modification, Phosphorylation, Sulfonation, Metastable atom activated dissociation, Cholecystokinin, Angiotensin II, Calcitonin

\section{Introduction}

$\mathrm{P}$ ost-translational modifications (PTMs) are chemical alterations of peptides and proteins following translation that usually provide a specific function such as signaling and activation. The need to unambiguously identify the type of PTM and the exact site of modification is critical to understand

Electronic supplementary material The online version of this article (doi:10.1007/s13361-011-0123-y) contains supplementary material, which is available to authorized users.

Correspondence to: Glen P. Jackson; e-mail: jacksong@ohio.edu the biological function and consequence. Phosphorylation and sulfonation are two examples of modifications that occur on carbohydrates and lipids in addition to proteins and peptides. One of the more common and well-studied modifications is phosphorylation: an addition of $-\mathrm{HPO}_{3}$ to tyrosine, threonine, and serine amino acid side chains. Phosphorylation detection is an integral part of proteomics because it is a major player in cell regulating mechanisms and signaling pathways, which control a wide variety of biological functions $[1,2]$. Tyrosine O-sulfonation is a less prevalent PTM, which occurs by a $-\mathrm{SO}_{3}$ transfer from phosphoadenosine phosphosulfate (PAPS) to the side chain of tyrosine residues. O-sulfonation is associated 
with protein transport and the regulation of proteinprotein interactions in the extracellular space [3].

For several decades mass spectrometry (MS) and tandem MS (MS/MS) have been used to identify and locate PTMs. Traditionally, the standard polarity and dissociation method used to analyze PTMs is positive ion mode and CID, respectively. Although there are thousands of articles on PTM analysis in positive ion mode within the past decade, fewer than $1 \%$ of the publications on PTM analysis have been dedicated to the use of negative ion mode. Despite the advances in PTM detection with MS/MS, there are some major drawbacks. Traditional CID preferentially cleaves side chain PTMs rather than the peptide backbone, thereby leading to ambiguous site determination. Even though phosphorylation modifications tend to be among the more stabile PTMs, Palumbo and Reid have shown evidence that under positive mode CID conditions, the phosphate groups can migrate to different amino acids and thereby result in ambiguous or unambiguously incorrect PTM site determination [4]. In addition, highly acidic peptides, such as those that contain phospho- and/or sulfo- groups, are difficult to observe in positive ion mode because of their low pKa values and poor ionization efficiency $[5,6]$. However, acidic peptides have been shown to ionize more readily in negative ion mode $[6,7]$. O-sulfonation, which is highly labile in positive ion mode, has shown to be slightly more stable in negative ion mode $[5,8]$. To date, the ability to routinely determine both phosphorylation and sulfonation modifications in anions, especially in highly acidic peptides and proteins, is not a routine mass spectrometry process.

To combat some of these issues, alternative dissociation techniques utilizing electron-radical chemistry such as electron capture and electron transfer dissociation (ECD and ETD) have become the preferred new way to sequence and analyze modified peptides and proteins [9-12]. These two methods have the ability to retain labile side chain modifications, while achieving extensive $\mathrm{N}-\mathrm{C}_{\alpha}$ bond cleavage of multiply charged cations. ECD and ETD have enhanced the ability for peptide sequencing and PTM site determination. To date, both methods have been successfully employed to determine phosphorylation [13, 14], glycosylation [15-17], and sulfonation [9, 10] modifications, among others. On the other hand, it has been recently shown that certain modifications, such as phosphorylation [18] and nitration [19-21], have hindered ECD and ETD sequence coverage and modification identification. Liu and Håkansson showed that highly acidic peptides with no basic amino acids produced complete loss of the sulfonated modification, thereby rendering site determination impossible by ECD [22]. To achieve improved ionization efficiency and modification retainment of highly acidic sulfonated peptides in ECD, charge enrichment through the formation of a divalent metal cation-peptide complex is necessary. In a similar experiment, Medzihradszky et al. investigated the labile nature of sulfonated peptides that contained only one basic amino acid employing both ETD and ECD [23]. Under native ETD and ECD conditions, $15 \%$ (in the best case) of the $-\mathrm{SO}_{3}$ modification retainment was observed for the multiply protonated cations [23]. The modification retainment improved to $73 \%$ (in the best case) when the peptides were complexed with $\mathrm{Na}^{+}$.

Traditionally, ECD and ETD were only applicable to multiply protonated species. Recently, new methods such as electron detachment dissociation (EDD), negative ETD (NETD), and negative-ion mode ECD (niECD) have been explored and applied to peptide anions. EDD, first applied to sulfonated di-anions in 2001 by Zubarev and co-workers, results in $\mathrm{C}_{\alpha}-\mathrm{C}$ peptide backbone cleavage by irradiating peptide anions with fast electrons $(>10 \mathrm{eV})$, which induces electron detachment from the peptides [24]. Similar to ECD, EDD requires costly FT-ICR instruments and has proven to be an inefficient process with interaction times on the scale of several seconds $[25,26]$. Coon et al. first demonstrated NETD or ion/ion reactions of xenon radical cations with polyanions resulting in $\mathrm{C}_{\alpha}-\mathrm{C}$ peptide backbone cleavage and neutral losses of $\mathrm{CO}_{2}$ and $\mathrm{HPO}_{3}$ from the product ions [27]. More recently, Huzarska et al. utilized a fluoranthene radical $\left(\mathrm{C}_{16} \mathrm{H}_{10}{ }^{+\bullet}\right)$ as the NETD reagent cation to induced EDD like fragmentation through the abstraction of an electron from the peptide anion by the reagent cation radical leaving the phosphorylation modification intact [28]. The difference in the degree of modification retainment in NETD is attributed to the difference in the recombination energy of the electron transfer process between the two reagent ions studied; 6.7$8.7 \mathrm{eV}$ for $\mathrm{Xe}^{+\bullet}$ and $2.5-4.5 \mathrm{eV}$ for the fluoranthene radical. Håkansson and co-workers unveiled impressive results exploring the ECD version for peptide anions termed negative-ion ECD at the 58th ASMS Conference on Mass Spectrometry and Allied Topics in Salt Lake City. However, little information is available to date [29, 30].

In the last five years, a new type of dissociation method has been introduced in rf ion trap instruments, which uses metastable atoms as the electron source or potential energy source to induce fragmentation. Extensive backbone cleavages in the form of a-, b-, c-, x-, y-, and z-type ions, and PTM retainment, is achieved through the interaction of isolated precursor ions with high or low kinetic energy beam of noble gas metastable atoms. Our research shows evidence that peptide dissociation occurs through two competing mechanisms, Penning ionization (PI) and charge reduction $[31,32]$. The later mechanism is very similar to ECD and ETD. PI occurs when the potential energy of the metastable atom is greater than the ionization potential of the biomolecular ion and has been well characterized for neutrals $[32,33]$. Metastable atoms are attracted to, and therefore most reactive with, regions of high electron density such as the lone pairs on nitrogen atoms or carbonyl oxygen atoms that are found throughout the peptide backbone and in amino acid side chains. Several papers have been devoted to this new type of research, characterizing multiply- and singly-charged cations, phosphorylated peptides, disulfide bond cleavage, isoleucine/leucine differentiation, and proline 
ring cleavage [31, 32, 34-37]. However, a small portion of these studies has been dedicated to the study of the interaction between metastable atoms and peptide anions $[31,32,37]$. To date, our group has been the only group to explore PTMs on peptide anions using MAD $[32,38]$.

In this present study, we investigate the ability of metastable atom-activated dissociation (MAD) to characterize acidic phosphorylated and sulfated peptide anions. The majority of fragment ions observed are a-/x-type ions, which correspond to $\mathrm{C}_{\alpha}-\mathrm{C}$ peptide backbone cleavages and is consistent with fragmentation observed in EDD. Additionally, we observed modest neutral losses of the phosphorylation and the sulfonation modification in comparison to CID. We compare the non-modified and modified 2- forms of the phosphorylated and sulfonated peptides. The PTMs did not appear to significantly hinder fragmentation, unlike nitrosylation [32]. Sequence coverage for the 3-forms of the phosphorylated and sulfonated peptides was superior to the 2 - charge states. Due to the unique chemistry of MAD, we were also able to acquire MS/MS from the highly acidic 1+ peptides that contain no basic amino acids. Complete peptide sequencing with unambiguous sulfonation site determination was also possible in positive ion mode; a unique feature of this technique.

\section{Experimental}

\section{Instrumentation}

All of the experiments were performed on a modified Bruker EsquireLC QIT MS (Bruker Daltonics, Bremen, Germany), which contains a $2 \mathrm{~mm}$ hole in the top portion of the ring electrode. The metastable atoms are generated by a modified Ion Tech FAB gun (P50 PSU; Teddington, UK), which is centered directly over the ring electrode hole. A deflection electrode positioned and mounted $1 \mathrm{~cm}$ from the exit orifice of the FAB gun deflects the majority of the free electrons and ions from entering the trap allowing neutral metastable atoms to enter the top hole of the ring electrode and interact with the isolated precursor ions in the center of the trap. One electrode on the deflection electrode was grounded and the other was biased to $+800 \mathrm{~V}$ using a high voltage power supply (EL 03R 15 L; Glassman High Voltage Inc., High Bridge, NJ, USA). To achieve a pulsed beam of high kinetic energy metastable atoms, the FAB gun anode power supply was replaced with a $10 \mathrm{kV}$ high voltage amplifier (ANT 10B10, Matsusada Precision Inc., Shiga, Japan). To pulse the metastable atom beam on only during the mass spectrometry duty cycle designated for dissociation, a trigger was taken from the Bruker EsquireLC corresponding to the start of ion accumulation and fed to a function/arbitrary waveform generator (33250A; Agilent, Santa Clara, CA). Complete instrumentation modification and pulse operation details has been previously described [31]. In order to decrease background ion signal, the FAB gun was turned on at a constant voltage between 5 and $6 \mathrm{~V}$ to 'pre-burn' away residual contaminants for 30 miutes prior to use each day. Although the lack of commercially available instrumentation may hinder the adoption of MAD by other laboratories, the hardware requirements necessary to achieve MAD are no more arduous than those required to achieve ETD. The main difference between adding MAD to a commercial instrument versus adding ETD is the requirement for a straight line-ofsight path for the metastable atoms to interact with the isolated ion cloud. ETD uses charge reagent anions, which can be steered using ion optics.

\section{Preparation of Peptides}

All of the following peptides were provided by Protea Biosciences Inc. (Morgantown, WV, USA): the phosphorylated and non-phosphorylated forms of angiotensin II, cholecystokin (10-20) and calcitonin (15-29); the sulfated and non-sulfated forms of cholecystokinin (26-33), leuenkephalin, and hirudin. Methanol (HPLC grade), ammonium hydroxide, and glacial acetic acid were purchased from Sigma-Aldrich (St. Louis, MO, USA). All peptides were reconstituted in a 1:1 mixture of methanol and water with either $1 \%$ acetic acid or $1 \%$ ammonium hydroxide (for the positive and negative ion mode, respectively) to provide solutions between 20 and $40 \mu \mathrm{M}$ of the individual peptides. Ultrahigh-purity helium (Airgas, Parkersburg, WV, USA) was further purified on-line with a noble gas purifier (HP2; VICI, Houston, TX, USA) before use in the FAB gun.

\section{Method}

An electronic syringe pump (BM-1000; Protea Bioscience Inc, Morgantown, WV, USA) was utilized to directly inject all peptides at a flow rate of $200 \mu \mathrm{L} / \mathrm{h}$. Depending on the modification and precursor charge state, the ion isolation windows varied between $m / z 1$ and 5. Ion accumulation was set to $15-25 \mathrm{~ms}$ to reach an ion target of 20,000. Depending on peptide and precursor charge state, the precursor ion intensity ranged from $8 \times 10^{4}$ to $4 \times 10^{5} \mathrm{AU}$. All fragment ion identifications and assignments were manually determined based on predicted fragmentation patterns and were within $m / z \pm 0.8$ of the expected product ions. Peaks intensities needed to meet at least three times the signal-to-noise ratio to be assigned. The 2-, 3-, and 1+ precursor ions were identified according to the expected $\mathrm{m} / \mathrm{z}$ value and the presence of an isotope envelope peak at $m / z+0.5$ and +0.3 , respectively. Setting a trapping voltage that corresponds to a low mass cutoff value above $\mathrm{m} / \mathrm{z}$ 200-400 (depending on charge state and ion mode) allowed for the removal of additional low mass background ions, which presumably result from Penning ionization of residual pump oil. In theory, the trapping $\mathrm{q}_{\mathrm{z}}$ value could be lowered in MAD to permit the detection of ions well below $\sim 1 / 3$ the precursor ion mass, which would overcome the low-mass-cut-off (LMCO) problem commonly experienced with CID. However, at present our LMCO is limited by the presence of background ions 
from residual contaminants, so we cannot yet take full advantage of the potential LMCO benefit of MAD.

\section{$C I D$}

All peptides were fragmented using the "SmartFrag" option in the Bruker EsquireLC NT 4.5 software, which applied a sweeping collisional energy starting at $20 \%$ and ending at $165 \%$ of a set value. The fragmentation time was set to $20 \mathrm{~ms}$ in all experiments. The collisional energy was set to 0.5-0.8 $\mathrm{V}$ depending on precursor mass and compound stability. CID MS/MS data was acquired in 1 min intervals, which consisted of approximately 30 scans.

\section{$M A D$}

All peptide precursor ions were exposed to a high kinetic energy beam of He metastable atoms with the FAB anode powered at $6 \mathrm{kV}$. Depending on the charge state of the precursor ion, the metastable atom beam was pulsed on for 75-150 ms while the isolated precursor ions were 'fragmented' at a collision energy of $0 \mathrm{~V}$ (i.e., no collisional activation). The pressure was measured with a GranvillePhillips Series 343 Bayard-Alpert vacuum gauge. The pressure in the vacuum chamber, outside the ion trap, was kept at 1.8 mbar (uncorrected) with helium leaking out of the trap and an additional 1.0 mbar (uncorrected) of the noble gas from the metastable atom source. MAD MS/MS data was acquired in 2 min intervals, which consisted of approximately 60 scans.

\section{Results and Discussion}

\section{Phosphorylated Peptide Anions}

Phosphorylated peptides had a precursor ion mass increase of 79 Da relative to the unmodified peptides, confirming the addition of a $-\mathrm{PO}_{3}$ group. Peptide sequence inserts in all figures omit those fragment ions that have undergone a neutral loss of the PTM modification. A lower case 'p' indicates the amino acid directly to the right is phosphorylated. Figure 1a and $b$ show MAD spectra of the non-phosphorylated and the phosphorylated 2- species of the peptide cholecystokinin (IKNLQSLDPpSH), respectively, which contains two serine residues with the tenth residue phosphorylated (Ser-10). PI causes oxidation or a change of +1 of the charge state of a given target molecule or ion. For cations, PI results in a charge increase, but for anions PI results in a less negative charge. For this reason, the PI products of 1charge states are most likely to be overall neutral in charge and not observable.

The main features of MAD of the 2- peptides in Figure 1a and $\mathrm{b}$ are the neutral losses from the oxidized species, $[\mathrm{M}-2 \mathrm{H}]^{-}$, corresponding to $\mathrm{CO}, \mathrm{CO}_{2}$, and $\left[\mathrm{CO}_{2}+\mathrm{H}_{2} \mathrm{O}\right]$ for both peptides and $\mathrm{HPO}_{3}$, and $\left[\mathrm{HPO}_{3}+\mathrm{H}_{2} \mathrm{O}\right]\left(\right.$ or $\left.\mathrm{H}_{3} \mathrm{PO}_{4}\right)$ for the phosphorylated peptide. Sequence coverage for both peptides is about $80 \%$. MAD of the non-phosphorylated precursor ion (Figure 1a) produced 12 distinct fragment ions in total, comprising of eight a-/x-type ions, two c-/z-, and two y-type ions. Similarly, MAD of the phosphorylated precursor ion (Figure 1b) produced eight a-/x-type ions, three c-/z-, and three $b-/ y$-type ions, totaling fourteen distinct fragment ions. The abundant observation of a-/x-type ions is consistent with observations with other types of negative electron transferring dissociation techniques such as EDD $[24,39]$ and NETD $[27,28]$. Only two fragment ions, $\mathrm{c}_{7}$ and $b_{8}$, underwent a neutral loss of the phosphorylation modification. However, both of these ions were also observed with the modification intact. MAD of the doubly-deprotonated cholecystokinin therefore enables unambiguous site determination to the Ser-10 residue. One notable difference between the modified and unmodified forms of the peptide is the relative abundance of the fragment ions $\mathrm{x}_{6}$ and $b_{8}$. These ions are among the most abundant ions in the mass spectrum of the phosphorylated form of cholecystokinin (Figure 1b), but are absent or in very low abundance in the nonmodified spectrum (Figure 1a).

Figure 1c and $\mathrm{d}$ show MAD spectra of the nonphosphorylated and the phosphorylated doubly-deprotonated species of the peptide calcitonin (DFNKFHpTFPQTAIGV), respectively, which contains two threonine residues with the seventh residue phosphorylated (Thr-7). Similar to cholecystokinin, the most dominant features in Figure $1 \mathrm{c}$ are the neutral losses of $\mathrm{H}_{2} \mathrm{O}, \mathrm{CO}_{2}$, and $\left[\mathrm{CO}_{2}+\mathrm{H}_{2} \mathrm{O}\right]$ from the oxidized species $[\mathrm{M}-2 \mathrm{H}]^{-}$. MAD of the non-phosphorylated calcitonin produced seventten distinct fragment ions with eleven of those ions comprised of a-/x-type ions. Conversely, when the same peptide is phosphorylated (Figure 1d) the number of fragment types slightly decreases and sequence coverage drops from $86 \%$ to $72 \%$. MAD of the 2- phosphorylated precursor ion (Figure 1d) produced fourteen product ions, with ten a-/x-type ions and three c-/z-type ions. The most abundant peaks in Figure 1d correspond to the oxidized species, $[\mathrm{M}-2 \mathrm{H}]^{-}$, and the neutral losses of $\mathrm{CO}_{2}$ and $\left[\mathrm{HPO}_{3}+\right.$ $\mathrm{H}_{2} \mathrm{O}$ ] (or $\mathrm{H}_{3} \mathrm{PO}_{4}$ ) from the oxidized species. Regardless of the slight decrease in sequence coverage, MAD still produces enough information to unambiguously determine the site of modification to the Thr-7 residue. Finally, MAD of the phosphorylated peptides produces very similar results in comparison to the non-phosphorylated peptide spectra such as: (1) ample backbone cleavage adjacent to and in the vicinity of the modification, and (2) abundant a-/x-type fragment ions showing consistent cleavage mechanism regardless of whether or not the phosphorylation group is present.

\section{MAD Versus CID}

We compared the fragmentation behavior of peptides through MAD and CID, which contained a phosphorylated 
(a)

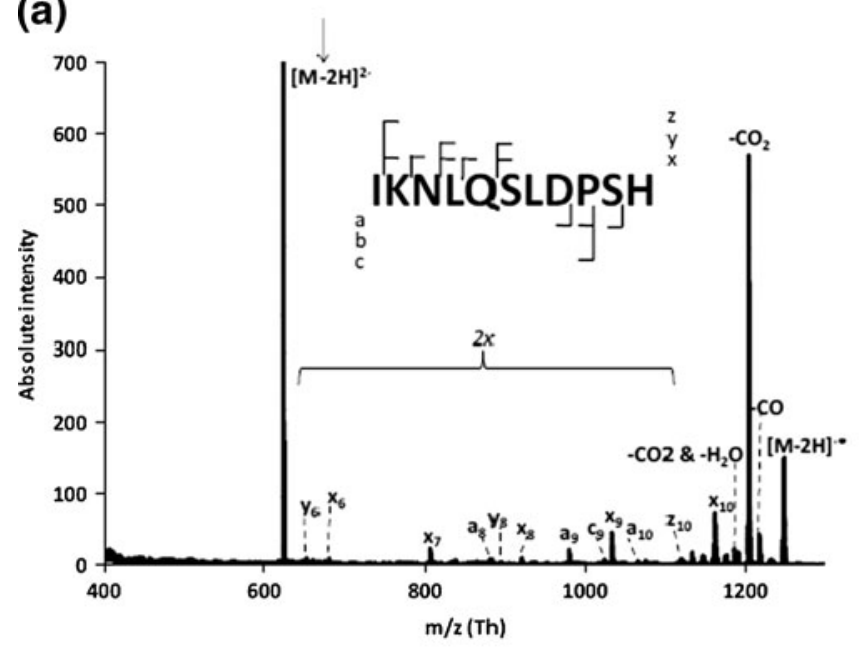

(c)

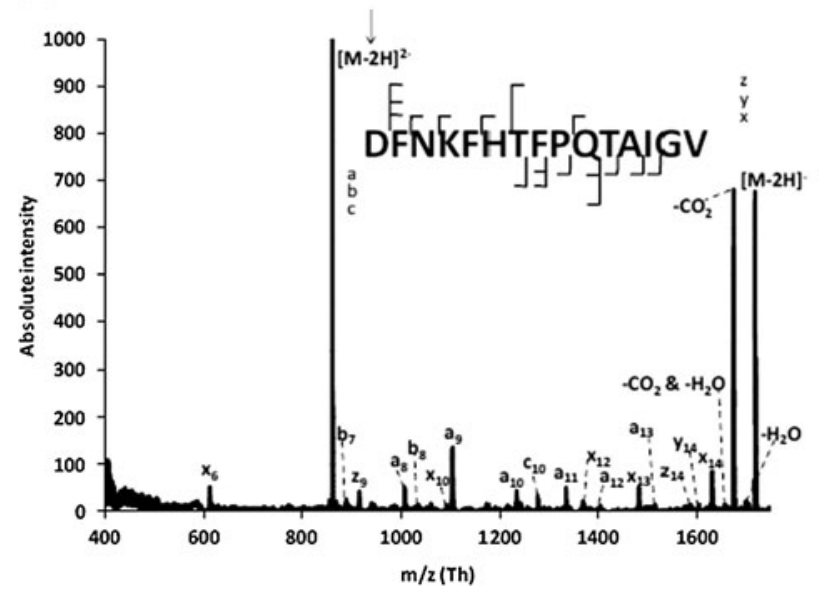

(b)

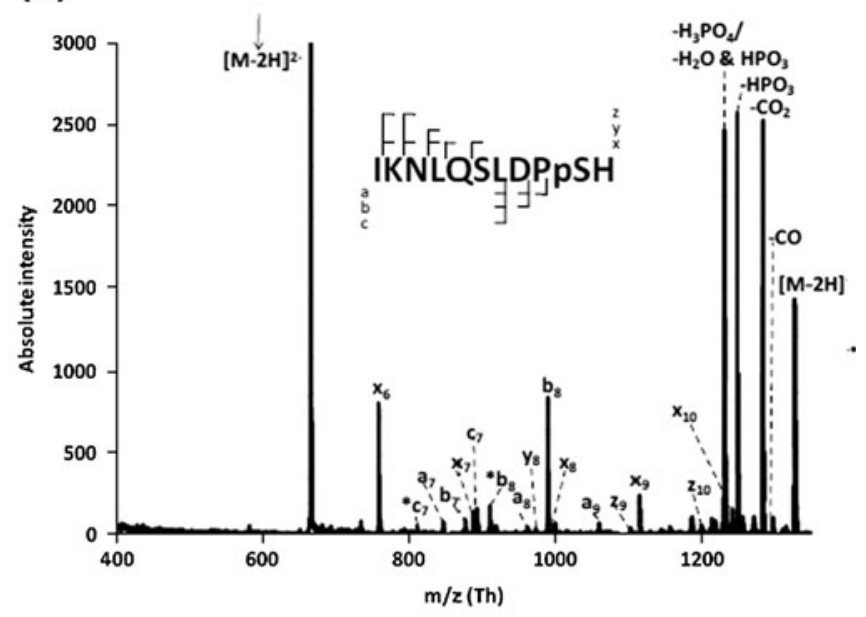

(d)

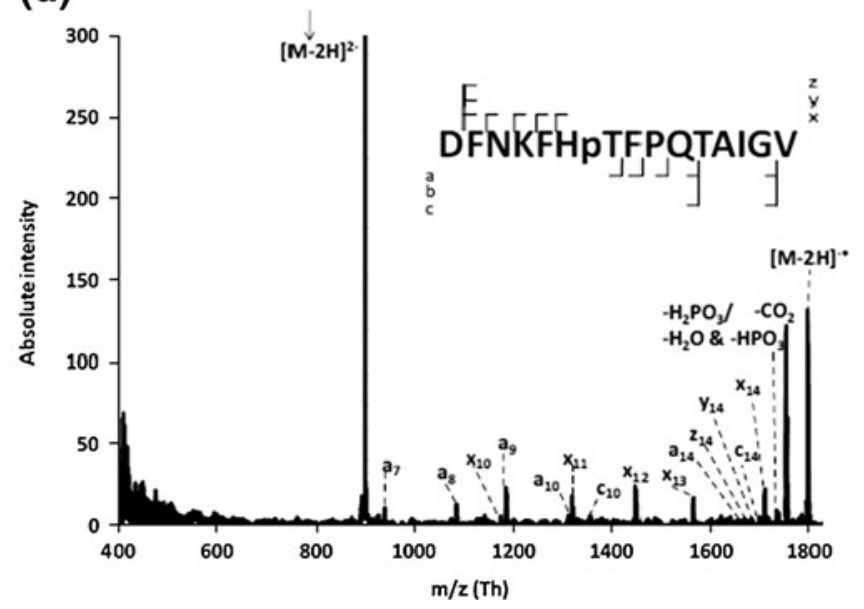

Figure 1. He MAD of cholecystokinin and calcitonin in the 2- charge state: (a) MAD of non-phosphorylated IKNLQSLDPSH, (b) MAD of phosphorylated IKNLQSLDPpSH, (c) MAD of non- phosphorylated DFNKFHTFPQTAIGV, and (d) MAD of phosphorylated DFNKFHPTFPQTAIGV. An arrow in each plot indicates the precursor. An asterisk $\left(^{*}\right)$ indicates fragments having lost the modification. Fragments that do not retain the modification are omitted from the peptides sequence inserts

tyrosine, threonine, or serine residue; the latter is described in Supplemental Figure 1S. MAD and CID mass spectra of the 3- form of calcitonin are compared in Figure $2 \mathrm{a}$ and $\mathrm{b}$. The MAD spectrum in Figure $2 \mathrm{a}$ allows for complete peptide sequencing and unambiguous site determination. CID only achieves $43 \%$ sequence coverage with four fragment ions showing modification loss. In this case, CID cannot distinguish which of the threonine residue contains the phosphorylation because there are no cleavages between the seventh and eleventh residues. In total, MAD produced twenty four fragment ions and CID produced eight fragment ions. Only four fragments were common to both methods (Supplemental Figure 2S). The main dissociation channels in CID are the neutral losses of $\mathrm{H}_{2} \mathrm{O}, \mathrm{HPO}_{3}$, and $\left[\mathrm{HPO}_{3}+\mathrm{H}_{2} \mathrm{O}\right]\left(\right.$ or $\left.\mathrm{H}_{3} \mathrm{PO}_{4}\right)$ from the precursor ions. In $\mathrm{MAD}$, however, $\mathrm{CO}_{2}$ is the only neutral loss observed from the two oxidized species, $[\mathrm{M}-3 \mathrm{H}]^{2-\bullet}$ and $[\mathrm{M}-3 \mathrm{H}]^{-\cdot \cdot}$.
Figure $2 \mathrm{c}$ and $\mathrm{d}$ show MAD and CID mass spectra of the 3 - form of angiotensin II. Again, MAD achieves significantly more sequence coverage in comparison to CID. MAD also retained the phosphorylation modification almost all of the time. Conversely, CID retains the modification only $25 \%$ of the time. CID produced only seven distinct fragments, whereas MAD produced thirteen. Again, only four fragment ions were common to both methods (Supplemental Figure 2S). MAD (Figure 2c) produced neutral losses of $\mathrm{CO}_{2}$ and $\left[\mathrm{HPO}_{3}+\mathrm{H}_{2} \mathrm{O}\right]$ (or $\mathrm{H}_{3} \mathrm{PO}_{4}$ ) from the two oxidized product ions, $[\mathrm{M}-3 \mathrm{H}]^{2-}$ and $[\mathrm{M}-3 \mathrm{H}]^{-*}$, but no neutral loss of the modification from the precursor ion was observed in CID (Figure 2d). However, CID of the 3- form of angiotensin II showed a dominant $\mathrm{y}_{4}$ ion and very little sequence information. The phosphate group was lost in most of the CID fragments. Similar results were also observed when comparing MAD and CID of the 2- charge state of phosphorylated cholecystokinin and calcitonin (Supplemental Figure 3S). 
(a)

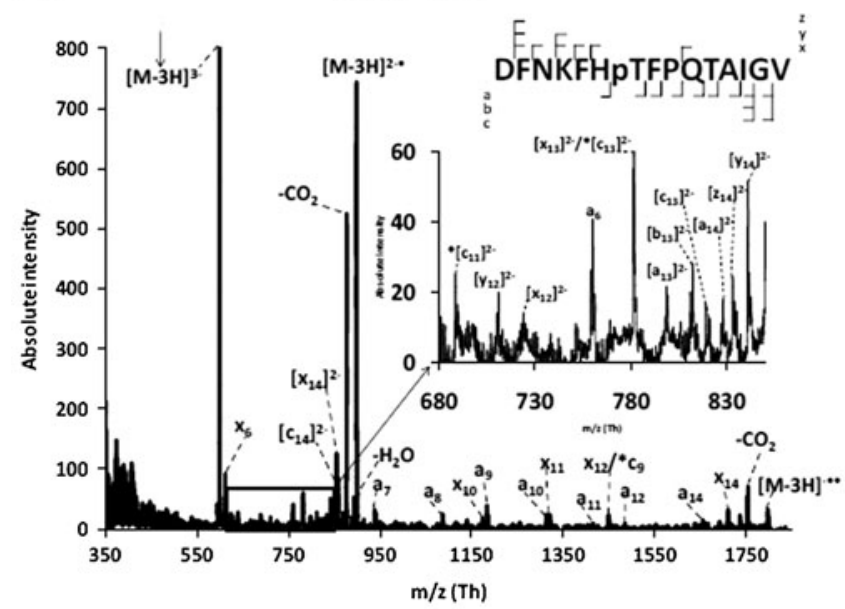

(c)

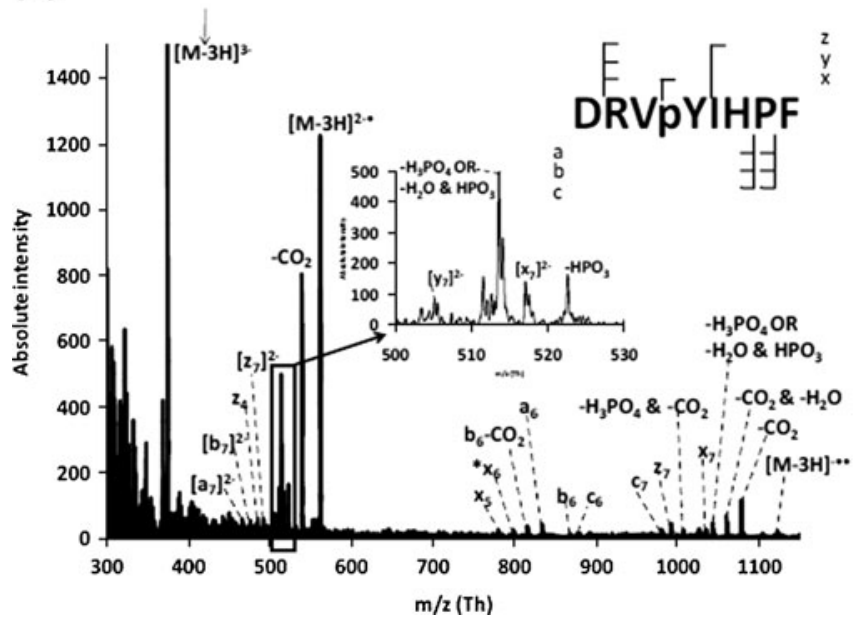

(b)

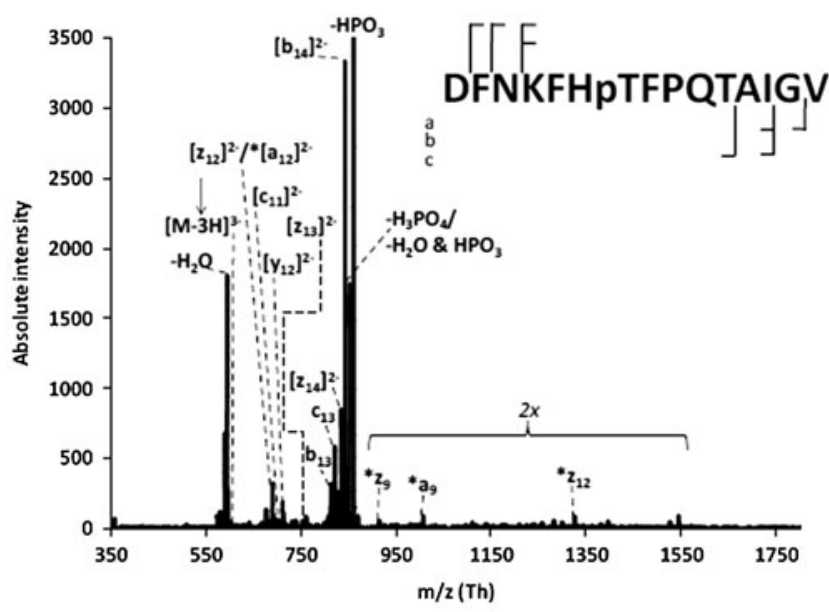

(d)

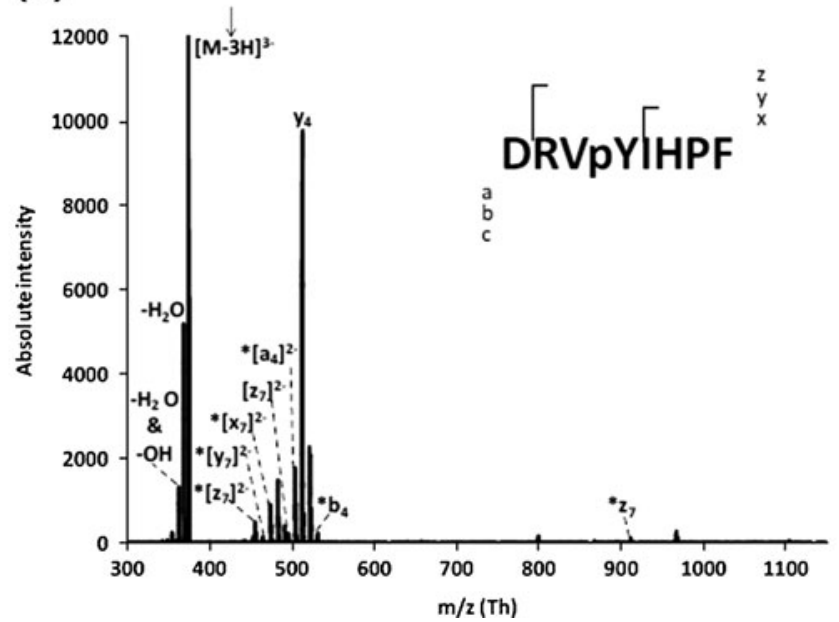

Figure 2. Comparison of He MAD and CID spectra of different phosphorylated peptides in the 3- charge state: (a) MAD and (b) CID of phosphorylated calcitonin (DFNKFHpTFPQTAIGV), (c) MAD and (d) CID of phosphorylated angiotensin II (DRVpYIHPF). An arrow in each plot indicates the precursor. An asterisk ( $\left.{ }^{\star}\right)$ indicates fragments having lost the modification. Fragments that do not retain the modification are omitted from the peptides sequence inserts

\section{Effect of Charge State on Peptide Backbone Cleavage}

Cleavages produced by MAD of the 2- and 3- forms of phosphorylated cholecystokinin, calcitonin, and angiotensin II are compared as Venn diagrams in Figure 3 and in Supplemental Table 1S. In general, sequence coverage increases only slightly as charge state increases. This observation is likely due to two reasons: (1) as additional charges are added to the precursor ion, the peptide unravels due to Coulombic repulsion and has a more open structure, and (2) the fragmentation energy (exothermicity) is different in Penning ionization of the 3 - versus the 2 - precursor. In our previous work with positive ion mode MAD, it was observed that the types of fragment ions produced by MAD were highly dependent on the charge state of the precursor ion [32]. In contrast to positive ion mode MAD, the types of fragment ions produced in negative ion mode MAD are apparently much less dependent on charge state. Between one-third and one-half of the total product ions observed in negative ion mode MAD are common to both the 2- and 3charge states (Figure 3a, b, and c).

Figure 4 and Supplemental Table $2 \mathrm{~S}$ compare the amounts and types of fragment ions MAD produces from the $2+$ and $2-$ forms of the phosphorylated peptides. In contrast to negative ion mode MAD, positive ion mode MAD produces more product ion types. Additionally, positive ion mode MAD enabled extensive fragmentation and modification retainment. The phosphorylation modification showed no deleterious effect on positive ion mode MAD backbone cleavage (Supplemental Figure 4S) unlike ECD of the 2+ phosphorylated species [18]. Compared to the respective $2+$ charge states, MAD of the 2 - charge state does not provide very many unique ions for two of the three peptides (cholecystokinin and 

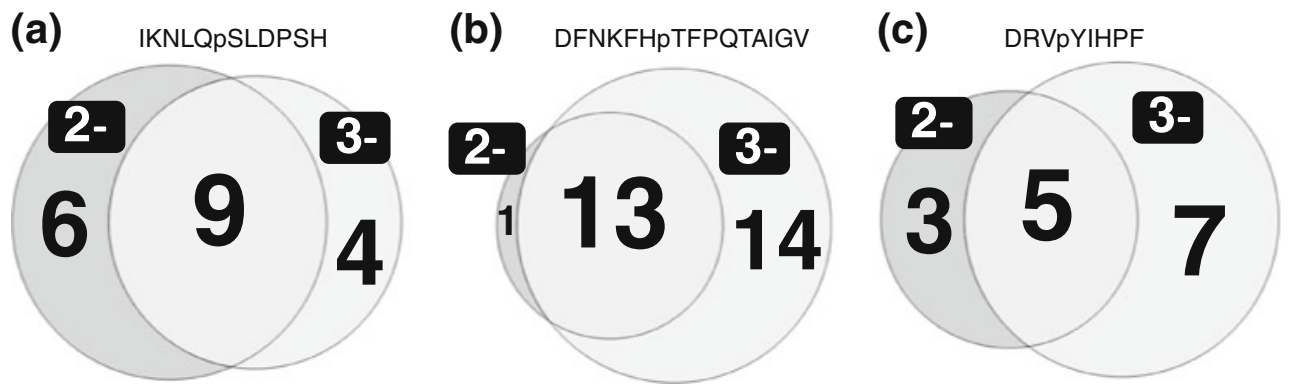

Figure 3. Venn diagrams comparing the number of fragment ion types produced after He MAD of the 2- and 3phosphorylated peptides: (a) cholecystokinin (IKNLQSLDPpSH), (b) calcitonin (DFNKFHpTFPQTAIGV), and (c) angiotensin II (DRVpYIHPF)

angiotensin II) but shows very unique fragments for the 2- form calcitonin. In general MAD of phosphorylated peptide anions is less dependent on charge state than positive mode and offers simpler mass spectra. Positive ion MAD generates more fragment ion types, but more complicated spectra. Each polarity has pros and cons and provides complementary fragment types.

\section{Sulfonated Peptide Anions}

The precursor ion masses of the various sulfonated peptides showed an increase of $79 \mathrm{Da}$ relative to the non-modified peptide in Figure $5 \mathrm{~b}$ and d, confirming the addition of an $\mathrm{SO}_{3}$ group. A lower case ' $\mathrm{s}$ ' indicates the amino acid directly to the right is modified. Figure $5 \mathrm{a}$ and $\mathrm{b}$ are the mass spectra of the 2- form of cholecystokinin, DsYMGWMDF, which corresponds to the non-sulfonated species and the sulfonated species dissociated with MAD. Again, the fragment ions that underwent a neutral loss of the modification are omitted in the peptide inserts in each of the figures. The non-sulfonated 2- precursor ions (Figure 5a) produced $100 \%$ sequence coverage with five a-/x-type ions, four c-/ z-type ions, and only one b-type ion. The most abundant dissociation channels are $\mathrm{C}-\mathrm{N}$ and $\mathrm{N}-\mathrm{C}_{\alpha}$ backbone cleavages between the methionine and aspartic acid residues, which correspond to the fragment ions $b_{6}$ and $c_{6}$.

MAD dissociation of the sulfonated precursor ions also produced five a-/x-type ions, three b-/y-type ions, and four c-/z-type ions as seen in Figure 5b. Comparing the degree and type of fragment ions observed between the nonsulfonated and the sulfonated precursor ions with MAD (Figure 5a and b), a small decrease (two ions) in sequence coverage is observed. The fragmentation behavior is similar to that observed by Zubarev and co-workers using EDD of the 2- form of sulfonated caerulein (pEQDsYTGWMDF), a very similar peptide to cholecystokinin [24]. Using EDD, Budnik et al. observed mainly a-, c-, and x-type ions [24]. However they observed more neutral losses of $\mathrm{CO}_{2}$ and $\mathrm{HSO}_{3}$ from the fragment ions than we observed here with MAD. Observation of additional backbone cleavage (Figure $5 \mathrm{~b}$ ) between the tryptophan and methionine, especially between the $\mathrm{C}_{\alpha}-\mathrm{C}$ bonds ( $\mathrm{a}_{5}$ ion), and the decrease in the abundance of the $b_{5}$ and $c_{5}$ ions suggests that sulfonation alters the preferred dissociation channels compared with the unmodified peptide.

Despite the differences in fragment ion types, an intense oxidized product ion, $[\mathrm{M}-2 \mathrm{H}]^{-}$, is observed in both Figure $5 \mathrm{a}$ and $\mathrm{b}$. In addition, the same neutral losses for the sulfonated on the non-sulfonated peptides are observed from the oxidized product ion, which correspond to $\mathrm{H}_{2} \mathrm{O}, \mathrm{CO}_{2}$, $\left[\mathrm{CO}_{2}+\mathrm{H}_{2} \mathrm{O}\right]$, and $\mathrm{HSO}_{3}$. Both peptides undergo a neutral loss of the aspartic acid side chain, $\left({ }^{\circ} \mathrm{CHO}_{2}\right)$ resulting in the $\mathrm{d}_{7}$ ion. Additionally, both peptides undergo neutral losses of the side chains of the methionine residue (indicated with a $\dagger$ in Figure $5 \mathrm{a}$ and $\mathrm{b}$ ) corresponding to a neutral loss of $74 \mathrm{Da}$ $\left({ }^{\circ} \mathrm{C}_{3} \mathrm{H}_{6} \mathrm{~S}\right)$ [40], and the tryptophan residue (indicated with a $\dagger$
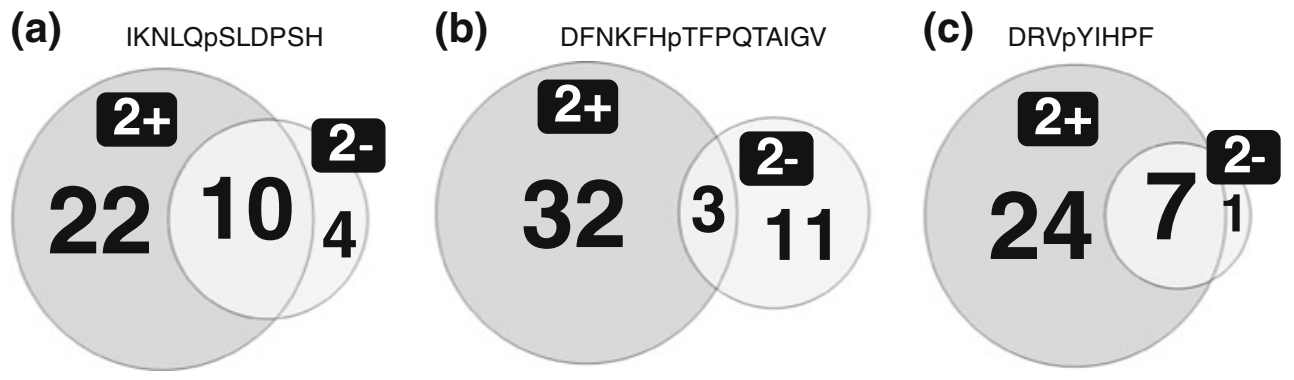

Figure 4. Venn diagrams comparing the number of fragment ion types produced after He MAD of the 2+ and 2phosphorylated peptides: (a) cholecystokinin (IKNLQSLDPpSH), (b) calcitonin (DFNKFHpTFPQTAIGV), and (c) angiotensin II (DRVpYIHPF) 

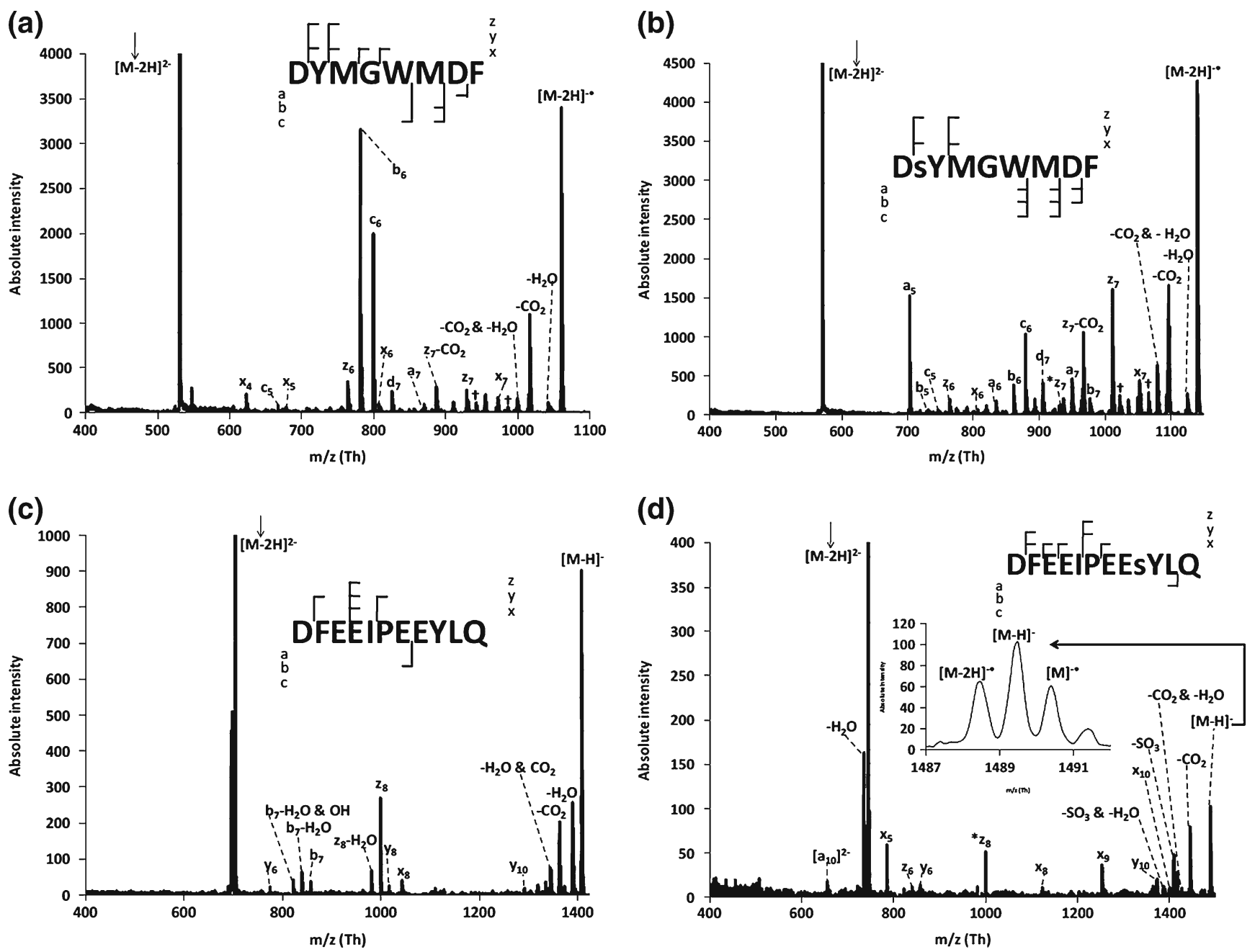

Figure 5. He MAD of sulfonated cholecystokinin and hirudin in the 2- charge state: (a) MAD of non-sulfonated DYMGWMDF, (b) MAD of sulfonated DsYMGWMDF, (c) MAD of non- sulfonated DFEEIPEEYLQ, and (d) MAD of sulfonated DFEEIPEEsYLQ. An arrow in each plot indicates the precursor. An asterisk $\left(^{*}\right)$ indicates fragments having lost the modification. Fragments that do not retain the modification are omitted from the peptides sequence inserts

in Figure 5a and b) corresponding to a neutral loss of $116 \mathrm{Da}$ $\left({ }^{\circ} \mathrm{C}_{8} \mathrm{H}_{6} \mathrm{~N}\right)$ from the oxidized product ion [41]. Comparing MAD and CID of the sulfonated species in Figure $5 \mathrm{~b}$ and Supplemental $5 \mathrm{~S}$, the sequence coverage is clearly superior using MAD. The sulfonation modification is lost only once when employing MAD, but many of the CID fragments lose the sulfonation group. The most abundant peak in the CID spectrum is the neutral loss of water from the precursor ion.

Figure $5 \mathrm{c}$ and $\mathrm{d}$ are MAD spectra of the $2-$ form of hirudin (DFEEIPEEsYLQ) in the non-sulfonated and sulfonated forms, respectively. Even though the sequence coverage increases from $40 \%$ to $60 \%$ from the non-sulfonated to the sulfonated species, the overall fragmentation efficiency is slightly less for the sulfonated peptide. The sulfonated peptide shows a much smaller charge reduced ion, [M $2 \mathrm{H}]^{-}$, and a larger $[\mathrm{M}-\mathrm{H}]^{-}$ion from a radical hydrogen loss. In addition, the fragment ions produced from the nonsulfonated species undergo several neutral losses, including $\cdot \mathrm{OH}, \mathrm{CO}_{2}$ and $\left[\mathrm{CO}_{2}+\mathrm{H}_{2} \mathrm{O}\right]$ from the oxidized product ion.
No water losses from the fragment ions are observed when the peptide is modified, but all the neutral losses besides $\cdot \mathrm{OH}$ are observed from the oxidized product ion (Figure $5 \mathrm{~d}$ ). When dissociating the sulfonated species with MAD (Figure 5d), five of the eight fragment ions are a-/x-type ions. CID of the sulfonated 2- form of hirudin (Supplemental Figure 5S) showed a large degree of neutral losses of water from the precursor and product ions. Additionally, CID achieves only three sequence ions and produces only two fragment ions that retain the modification.

MAD and CID fragmentation of the sulfonated 3peptides of cholecystokinin and hirudin are compared in Figure 6. MAD dissociation of the sulfonated cholecystokinin (Figure 6a) produced five a-/x-type ions, three b-type ions, and four c-/z-type ions, which achieved $72 \%$ sequence coverage and no modification losses, a total of thirteen product ions. Additionally, MAD produced a $\mathrm{d}_{7}$ ion, which results from a side chain cleavage of the aspartic acid residue from the $\mathrm{a}_{7}$ ion. Two oxidized species, $[\mathrm{M}-3 \mathrm{H}]^{2-}$ and $[\mathrm{M}-$ 
(a)

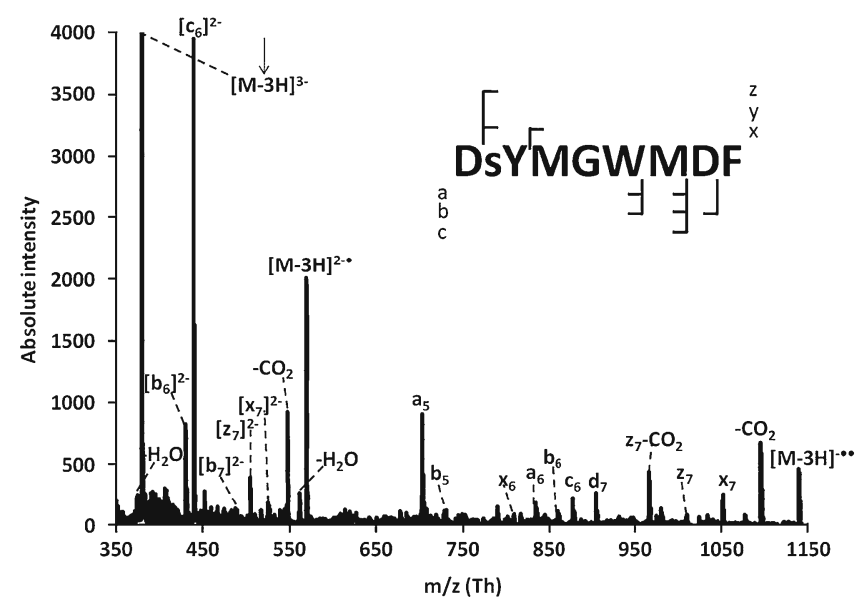

(c)

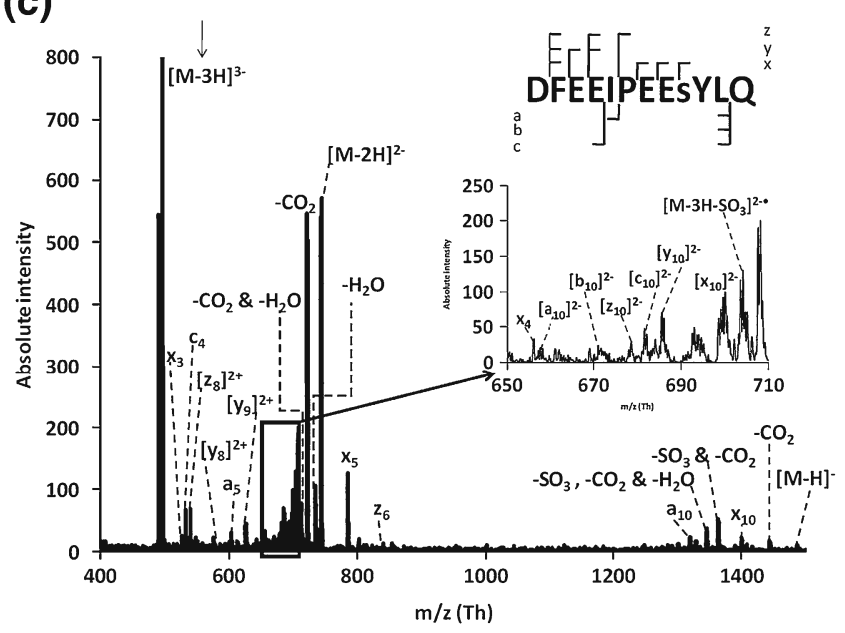

(b)

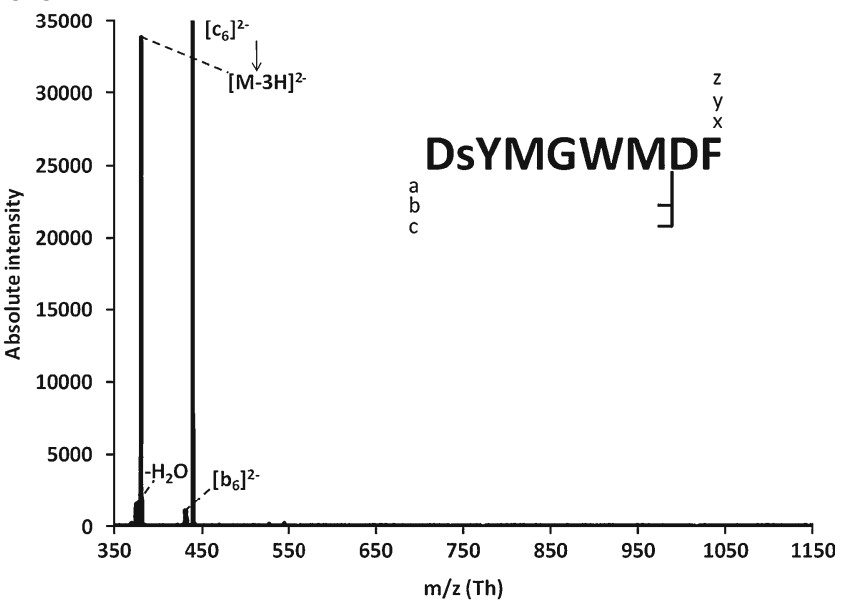

(d)

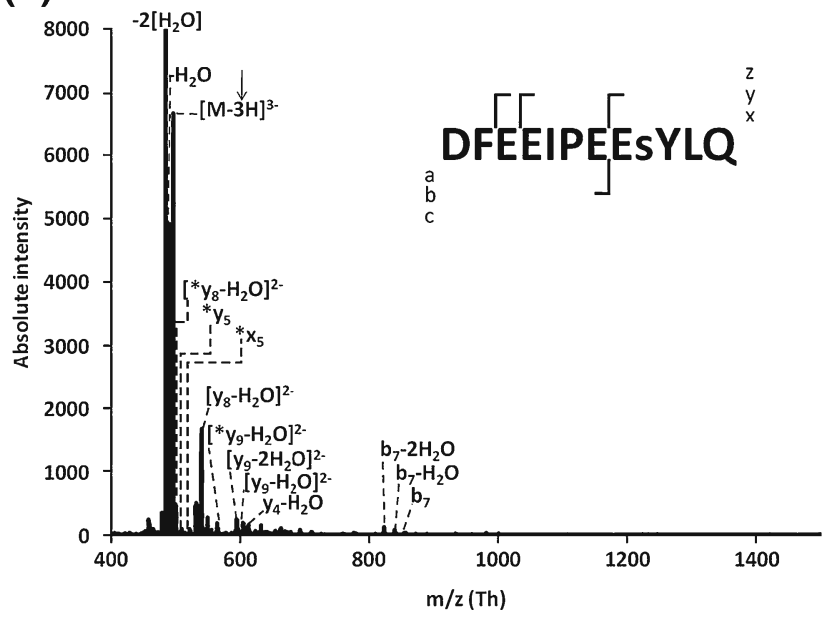

Figure 6. Tandem mass spectra comparing He MAD and CID of sulfonated cholecystokinin and hirudin in the 3- charge state: (a) MAD of sulfonated DsYMGWMDF, (b) CID of sulfonated DsYMGWMDF, (c) MAD of sulfonated DFEEIPEEsYLQ, and (d) CID of sulfonated DFEEIPEEsYLQ. An arrow in each plot indicates the precursor. An asterisk $\left(^{*}\right)$ indicates fragments having lost the modification. Fragments that do not retain the modification are omitted from the peptides sequence inserts

$3 \mathrm{H}]^{-\bullet}$, are observed in Figure 6a, presumably resulting from two consecutive Penning ionization reactions. CID (Figure $6 \mathrm{~b}$ ) only produced one $\mathrm{b}$ ion and one $\mathrm{c}$ ion and almost no structural information. When the 2 - form of the non-sulfonated cholecystokinin is fragmented with MAD the $\mathrm{b}_{6}$ and $\mathrm{c}_{6}$ ions are very dominant (Figure 5a). When sulfonated at the tyrosine residue (Tyr-2) the $\mathrm{b}_{6}$ and $\mathrm{c}_{6}$ ions are dramatically reduced and the $\mathrm{a}_{5}$ ion is more dominant (Figure $5 b$ ). When the same sulfonated peptide is fragmented in 3- charge state with MAD (Figure 6a) the $b_{6}$ and $\mathrm{c}_{6}$ ions return as the most abundant fragments. CID of either the 2- or 3- charge state of the sulfonated peptide also produces the $b_{6}$ and $c_{6}$ in high abundance indicating that these two fragments have a relatively low activation energies and are highly favorable. These observations indicate that when cholecystokinin is not sulfonated the charge has a high propensity for the aspartic acid at the Asp-7 position and promotes backbone cleavage adjacent to this residue. When the peptide is sulfonated at Tyr-2, an additional site of potential deprotonation is created. Because of the highly acidic nature of the modification, the charge has a higher probability of residing on the sulfate group than the aspartic acid residue. The remaining charge is then located on one of the three remaining acidic sites. The effect of sulfonation is to reduce the probability of locating the charge at the Asp-7 position.

When the peptide is in the 3-charge state there is an additional charge, so the probability of deprotonation at the Asp-7 position increases. The increased probability of charge-directed fragmentation adjacent to the aspartic acid residue for the 3- precursor explains the increased relative abundance of the $b_{6}$ and $c_{6}$ ions relative to the 2- charge state. This suggests that the specific site of deprotonation, such as acidic acids and the sulfate moiety, appears to influence the favorability for site-specific cleavages.

MAD dissociation of the sulfonated 3- hirudin (Figure 6c) achieved $90 \%$ sequence coverage with no observed loss of the modification. CID of the same 3 - precursor provided $30 \%$ coverage and only three fragment ions that retained the 
modification (Figure 6d). MAD produced seventeen fragment ions in total with eight a-/x-type ions, four b-/y-type ions, and five c-/z-type ions. Two oxidized species are observed in Figure $6 \mathrm{c}$ resulting from consecutive Penning ionization reactions. However the most intense peaks within the isotopic distributions, $[\mathrm{M}-2 \mathrm{H}]^{2-}$ and $[\mathrm{M}-\mathrm{H}]^{-}$, which correspond to neutral losses of $\mathrm{H}^{*}$ and $2 \mathrm{H}^{*}$ or $\mathrm{H}_{2}$, respectively, which is a common observation in EDD that is similarly indicated by electron abstraction [39]. CID of the 3- form of hirudin produced six distinct fragment ions, all cleaving the weaker $\mathrm{C}-$ $\mathrm{N}$ peptide backbone bonds. Dissociation with CID produced abundant water loss from the precursor and product ions, which is not observed with MAD. With the limited number of peptides studied to date, the 3- charge states seem to offer slightly better sequencing and modification retainment than the 2 - charge states.

\section{Sulfonated Peptide Cations: 1+ Charge State}

Unlike ECD and ETD, MAD does not utilize a charged reagent to initiate electron transferring/abstraction processes. Therefore MAD has the unique ability to dissociate $1+$ species and perform analysis of peptides without basic amino acids, such as DsYMGWMDF and sYGGFL (Figure 7). The mass spectrum in Figure 7a is of 1+ cholecystokinin, which contains no basic amino acids and a sulfonated tyrosine residue at the Tyr-2 position. Therefore, the only places for protonation are at the N-terminus or along the peptide backbone. The oxidized product ion, $[\mathrm{M}+\mathrm{H}]^{2+\bullet}$, along with three $2+$ product ions provides evidence for the mechanism of Penning ionization. Extensive backbone cleavage in the form of $\mathrm{C}_{\alpha}-\mathrm{C}, \mathrm{C}-\mathrm{N}$, and $\mathrm{N}-\mathrm{C}_{\alpha}$ bond cleavages produced $100 \%$ sequence coverage with $\mathrm{He}$ MAD. In total, 23 distinct fragment ions were observed with nine c-/z-type ions, nine b-/y-type ion, and five a-/x-type ions. The intense peaks at $m / z 331$ and 359, which correspond to the $a_{2}$ and $b_{2}$ fragment ions, suggest that the main dissociation channel is adjacent to the sulfonated tyrosine residue. In addition, neutral losses from the side chain of the methionine residue are observed twice. One such loss is the methionine side chain from the oxidized product ion, corresponding to neutral loss of $74 \mathrm{Da}$ (indicated with $\mathrm{a} \dagger$ in Figure 7). This loss is similarly seen in negative ion mode MAD. The second neutral loss is observed from the $z_{5}$ ion, corresponding to a neutral loss of a ${ }^{\circ} \mathrm{C}_{2} \mathrm{H}_{5} \mathrm{~S}$, thereby producing the $\mathrm{w}_{5}$ ion. These neutral losses from the methionine residue are also observed in ECD [40, 41]. Several neutral losses from the precursor ions are observed in the form of ${ }^{\circ} \mathrm{OH}, 2\left[^{\circ} \mathrm{OH}\right]$, and $\mathrm{CO}_{2}$. The majority of fragment ions retained the labile sulfate modification, which allowed for unambiguous site determination. These results are in stark contrast to the complete sulfonate loss that Liu and Håkansson 2 observed when employing ECD on 2+ cholecystokinin, which was attributed to the promotion of a mobile proton on the peptide backbone amide nitrogens [22]. Modification retainment improved in ECD when the cholecystokinin was complexed with different divalent metal cations. However $100 \%$ sequence
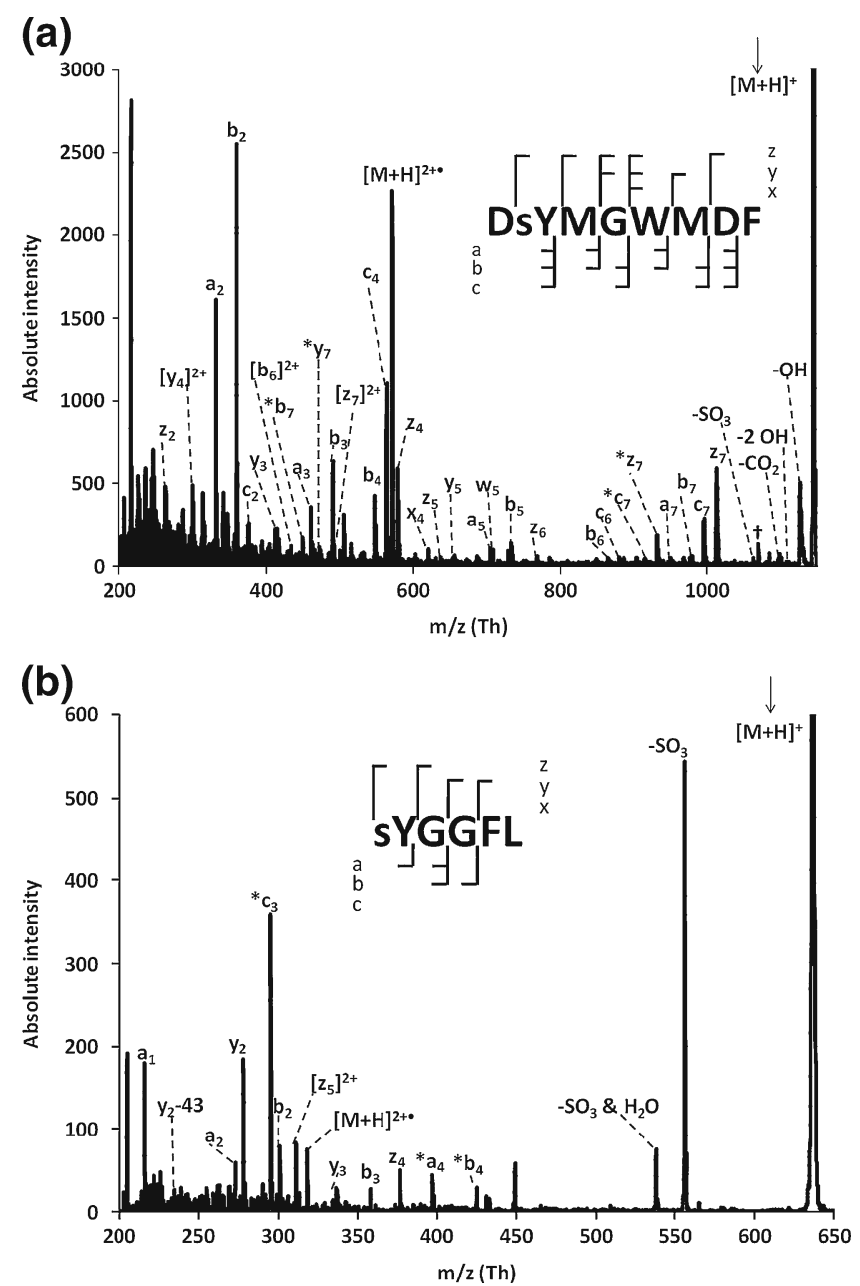

Figure 7. He MAD spectra of sulfonated angiotensin II and leucine enkephalin in the 1+ charge state: (a) DsYMGWMDF and (b) sYGGFL. An arrow in each plot indicates the precursor. An asterisk $\left({ }^{*}\right)$ indicates fragments having lost the modification. Fragments that do not retain the modification are omitted from the peptides sequence inserts

coverage was not achieved and significant neutral losses of $\mathrm{NH}_{3}, \mathrm{CO}_{2}, \mathrm{H}_{2} \mathrm{O}, \mathrm{CH}_{3} \mathrm{CO}_{2} \mathrm{H}$, and $\mathrm{H}^{\bullet}$ were observed.

Figure $7 \mathrm{~b}$ shows a MAD spectrum of $1+$ leucineenkephalin with a sulfonated tyrosine residue at the Tyr-1 position and no basic amino acids. Including those fragment ions that have undergone a neutral loss of the modification, cleavage between each amino acid is achieved. A total of twelve fragment ions are observed with the majority consisting of b-/y-type ions. A small PI peak, $[\mathrm{M}+\mathrm{H}]^{2+\bullet}$, is observed, as well as one $2+$ product ion, $\left[\mathrm{z}_{5}\right]^{2+}$. The $\mathrm{y}_{2}$ product ion also undergoes a neutral loss of $43 \mathrm{Da}$. This ion allows for the differentiation of leucine over isoleucine because of the different neutral losses of the sides chains: $43 \mathrm{Da}$ for leucine $\left({ }^{\circ} \mathrm{CH}\left(\mathrm{CH}_{3}\right)_{2}\right)$ and $29 \mathrm{Da}$ for isoleucine $\left({ }^{\circ} \mathrm{CH}_{2} \mathrm{CH}_{3}\right)$ [42]. The major dissociation channel for leucine enkephalin appears to be the loss of the sulfonation group from the precursor ion. Only three of the twelve fragment 
ions experienced a neutral loss of the sulfonation. These fragments allowed for unambiguous PTM site determination. Unlike ECD, MAD does not require additional charge enrichment steps to improve ionization efficiency or to stabilize the modification. MAD of the $1+$ charge state of the sulfated cholecystokinin appears superior to the 2- and 3charge states even though the same method of activation, PI, is responsible in all three cases. Clearly, the fragmentation methods and the fragmentation ions resulting from PI are influenced to some degree by the overall charge and conformation of the precursor ion.

\section{Conclusion}

Phosphorylated and sulfonated peptides are highly important modifications that need routine MS/MS analysis. The majority of today's analysis techniques rely on positive ion mode polarity mass spectrometry coupled with CID for modification identification. However, these techniques are not always optimal due to the modifications' acidic nature and their tendency to be cleaved during collisional activation. Coupling negative ion mode with MAD is a viable alternative to the traditional MS/MS analysis because sulfonated and phosphorylated peptides ionize more readily in the negative mode and MAD retains the more labile modifications. Ample backbone cleavage was observed for the 2- and 3- forms of phosphorylated and sulfonated peptides, allowing for peptide sequencing. Regardless of which amino acid is phosphorylated (serine, threonine, or tyrosine), MAD achieves unambiguous modification site determination even when numerous potential phosphorylation sites exist. In addition, MAD has the unique ability to dissociate $1+$ peptides without the need of charge enrichment steps or metal adduct formation. MAD offers a complementary alternative to traditional collisional or other electron mediated dissociation techniques.

\section{Acknowledgments}

The authors thank Protea Biosciences Inc. for the donation of the all the peptides used in this study and syringe pump. They also thank The Ohio State University and Kari Green-Church for the donation of the Bruker Esquire MS. The authors acknowledge Bascom French, their machine shop specialist, and Paul Schmittauer, their electronics specialist, for their work. This work was funded by NSF BIO 0649757 and an NSF CAREER Award CHE 0745590.

\section{References}

1. Garcia, B.A., Shabanowitz, J., Hunt, D.F.: Analysis of Protein Phosphorylation by Mass Spectrometry. Methods 35, 256-264 (2005)

2. Salih, E.: Phosphoproteomics by Mass Spectrometry and Classical Protein Chemistry Approaches. Mass Spectrom. Rev. 24, 828-846 (2005)
3. Yu, Y.H., Hoffhines, A.J., Moore, K.L., Leary, J.A.: Determination of the Sites of Tyrosine O-Sulfation in Peptides and Proteins. Nat. Methods 4, 583-588 (2007)

4. Palumbo, A.M., Reid, G.E.: Evaluation of Gas-Phase Rearrangement and Competing Fragmentation Reactions on Protein Phosphorylation Site Assignment Using Collision Induced Dissociation-MS/MS and MS $^{3}$. Anal. Chem. 80, 9735-9747 (2008)

5. Edelson-Averbukh, M., Pipkorn, R., Lehmann, W.D.: Phosphate Group-Driven Fragmentation of Multiply Charged Phosphopeptide Anions. Improved Recognition of Peptides Phosphorylated at Serine, Threonine, or Tyrosine by Negative Ion Electrospray Tandem Mass Spectrometry. Anal. Chem. 78, 1249-1256 (2006)

6. Janek, K., Wenschuh, H., Bienert, M., Krause, E.: Phosphopeptide Analysis by Positive and Negative Ion Matrix-Assisted Laser Desorption/Ionization Mass Spectrometry. Rapid Commun. Mass Spectrom. 15, 1593-1599 (2001)

7. Gunawardena, H.P., Emory, J.F., McLuckey, S.A.: Phosphopeptide Anion Characterization Via Sequential Charge Inversion and ElectronTransfer Dissociation. Anal. Chem. 78, 3788-3793 (2006)

8. Salek, M., Costagliola, S., Lehmann, W.D.: Protein Tyrosine-OSulfation Analysis by Exhaustive Product Ion Scanning with Minimum Collision Offset in a NanoESI Q-TOF Tandem Mass Spectrometer. Anal. Chem. 76, 5136-5142 (2004)

9. Kelleher, R.L., Zubarev, R.A., Bush, K., Furie, B., Furie, B.C., McLafferty, F.W., Walsh, C.T.: Localization of Labile Posttranslational Modifications by Electron Capture Dissociation: The Case of Gamma-Carboxyglutamic Acid. Anal. Chem. 71, 4250-4253 (1999)

10. Mikesh, L.M., Ueberheide, B., Chi, A., Coon, J.J., Syka, J.E.P., Shabanowitz, J., Hunt, D.F.: The Utility of ETD Mass Spectrometry in Proteomic Analysis. Biochem. Biophys. Acta 1764, 1811-1822 (2006)

11. Syka, J.E.P., Coon, J.J., Schroeder, M.J., Shabanowitz, J., Hunt, D.F.: Peptide and Protein Sequence Analysis by Electron Transfer Dissociation Mass Spectrometry. Proc. Natl. Acad. Sci. U.S.A. 101, 9528-9533 (2004)

12. Zubarev, R.A., Kelleher, N.L., McLafferty, F.W.: Electron Capture Dissociation of Multiply Charged Protein Cations. A Nonergodic Process. J. Am. Chem. Soc. 120, 3265-3266 (1998)

13. Chi, A., Huttenhower, C., Geer, L.Y., Coon, J.J., Syka, J.E.P., Bai, D. L., Shabanowitz, J., Burke, D.J., Troyanskaya, O.G., Hunt, D.F.: Analysis of Phosphorylation Sites on Proteins from Saccharomyces Cerevisiae by Electron Transfer Dissociation (ETD) Mass Spectrometry. Proc. Nat. Acad. Sci. U.S.A. 104, 2193-2198 (2007)

14. Stensballe, A., Jensen, O.N., Olsen, J.V., Haselmann, K.F., Zubarev, R. A.: Electron Capture Dissociation of Singly and Multiply Phosphorylated Peptides. Rapid Commun. Mass Spectrom. 14, 1793-1800 (2000)

15. Håkansson, K., Cooper, H.J., Emmett, M.R., Costello, C.E., Marshall, A.G., Nilsson, C.L.: Electron Capture Dissociation and Infrared Multiphoton Dissociation MS/MS of an N-Glycosylated Tryptic Peptide to Yield Complementary Sequence Information. Anal. Chem. 73, 4530$4536(2001)$

16. Mirgorodskaya, E., Roepstorff, P., Zubarev, R.A.: Localization of OGlycosylation Sites in Peptides by Electron Capture Dissociation in a Fourier Transform Mass Spectrometer. Anal. Chem. 71, 4431-4436 (1999)

17. Zhang, Q.B., Frolov, A., Tang, N., Hoffmann, R., van de Goor, T., Metz, T.O., Smith, R.D.: Application of Electron Transfer Dissociation Mass Spectrometry in Analyses of Non-Enzymatically Glycated Peptides. Rapid Commun. Mass Spectrom. 21, 661-666 (2007)

18. Creese, A.J., Cooper, H.J.: The Effect of Phosphorylation on the Electron Capture Dissociation of Peptide Ions. J. Am. Soc. Mass Spectrom. 19, 1263-1274 (2008)

19. Jones, A.W., Mikhailov, V.A., Iniesta, J., Cooper, H.J.: Electron Capture Dissociation Mass Spectrometry of Tyrosine Nitrated Peptides. J. Am. Soc. Mass Spectrom. 21, 268-277 (2010)

20. Sohn, C.H., Chung, C.K., Yin, S., Ramachandran, P., Loo, J.A., Beauchamp, J.L.: Probing the Mechanism of Electron Capture and Electron Transfer Dissociation Using Tags with Variable Electron Affinity. J. Am. Chem. Soc. 131, 5444-5459 (2009)

21. Tureček, F.: Electron Predators are Hydrogen Atom Traps. Effects of Aryl Groups on the $\mathrm{N}-\mathrm{C} \alpha$ Bond Dissociations of Peptide Radicals. $J$. Mass Spectrom. 45, 1280-1290 (2010) 
22. Liu, H., Håkansson, K.: Electron Capture Dissociation of Tyrosine OSulfated Peptides Complexed with Divalent Metal Cations. Anal. Chem. 78, 7570-7576 (2006)

23. Medzihradszky, K.F., Guan, S., Maltby, D.A., Burlingame, A.L.: Sulfopeptide Fragmentation in Electron-Capture and ElectronTransfer Dissociation. J. Am. Soc. Mass Spectrom. 18, 1617-1624 (2007)

24. Budnik, B.A., Haselmann, K.F., Zubarev, R.A.: Electron Detachment Dissociation of Peptide Di-Anions: An Electron-Hole Recombination Phenomenon. Chem. Phys. Lett. 342, 299-302 (2001)

25. Kweon, H.K., Håkansson, K.: Metal Oxide-Based Enrichment Combined with Gas-Phase Ion-Electron Reactions for Improved Mass Spectrometric Characterization of Protein Phosphorylation. J. Proteome Res. 7, 749-755 (2008)

26. Wolff, J.J., Amster, I.J., Chi, L.L., Linhardt, R.J.: Electron Detachment Dissociation of Glycosaminoglycan Tetrasaccharides. J. Am. Soc. Mass Spectrom. 18, 234-244 (2007)

27. Coon, J.J., Shabanowitz, J., Hunt, D.F., Syka, J.E.P.: Electron Transfer Dissociation of Peptide Anions. J. Am. Soc. Mass Spectrom. 16, 880$882(2005)$

28. Huzarska, M., Ugalde, I., Kaplan, D.A., Hartmer, R., Easterling, M.L., Polfer, N.C.: Negative Electron Transfer Dissociation of Deprotonated Phosphopeptide Anions: Choice of Radical Cation Reagent and Competition between Electron and Proton Transfer. Anal. Chem. 82, 2873-2878 (2010)

29. Hersberger, K.E., Brant, A., Wang, N., Håkansson, K.: Proceedings of the 58th ASMS Conference on Mass Spectormetry and Allied Topics. Salt Lake City, UT (May, 2010)

30. Yoo, H.J., Zhuang, S., Wang, N., Håkansson, K.: Proceedings of the 58th ASMS Conference on Mass Spectormetry and Allied Topics. Salt Lake City, UT (May, 2010)

31. Cook, S.L., Collin, O.L., Jackson, G.P.: Metastable Atom-Activated Dissociation Mass Spectrometry: Leucine/Isoleucine Differentiation and Ring Cleavage of Proline Residues. J. Mass Spectrom. 44, 1211-1223 (2009)
32. Cook, S.L., Jackson, G.P.: Characterization of Tyrosine Nitration and Cysteine Nitrosylation Modifications by Metastable Atom-Activation Dissociation Mass Spectrometry. J Am. Soc. Mass Spectrom. 22, 221$232(2011)$

33. Siska, P.E.: Molecular-Beam Studies of Penning Ionization. Rev. Mod. Phys. 65, 337-412 (1993)

34. Berkout, V.D.: Fragmentation of Protonated Peptide Ions Via Interaction with Metastable Atoms. Anal. Chem. 78, 3055-3061 (2006)

35. Berkout, V.D.: Fragmentation of Singly Protonated Peptides Via Interaction with Metastable Rare Gas Atoms. Anal. Chem. 81, 725731 (2009)

36. Berkout, V.D., Doroshenko, V.M.: Fragmentation of Phosphorylated and Singly Charged Peptide Ions Via Interaction with Metastable Atoms. Int. J. Mass Spectrom. 278, 150-157 (2008)

37. Misharin, A.S., Silivra, O.A., Kjeldsen, F., Zubarev, R.A.: Dissociation of Peptide Ions by Fast Atom Bombardment in a Quadrupole Ion Trap. Rapid Commun. Mass Spectrom. 19, 2163-2171 (2005)

38. Cook, S.L., Jackson, G.P.: Proceedings of the 58th ASMS Conference on Mass Spectormetry and Allied Topics. Salt Lake City, UT (May, 2010)

39. Kjeldsen, F., Silivra, O.A., Ivonin, I.A., Haselmann, K.F., Gorshkov, M., Zubarev, R.A.: $\mathrm{C} \alpha-\mathrm{C}$ Backbone Fragmentation Dominates in Electron Detachment Dissociation of Gas-Phase Polypeptide Polyanions. Chem. Eur. J. 11, 1803-1812 (2005)

40. Cooper, H.J., Hudgins, R.R., Håkansson, K., Marshall, A.G.: Characterization of Amino Acid Side Chain Losses in Electron Capture Dissociation. J. Am. Soc. Mass Spectrom. 13, 241-249 (2002)

41. Savitski, M.M., Nielsen, M.L., Zubarev, R.A.: Side-Chain Losses in Electron Capture Dissociation to Improve Peptide Identification. Anal. Chem. 79, 2296-2302 (2007)

42. Kjeldsen, F., Haselmann, K.F., Sorensen, E.S., Zubarev, R.A.: Distinguishing of Ile/Leu Amino Acid Residues in the PP3 Protein by (Hot) Electron Capture Dissociation in Fourier Transform Ion Cyclotron Resonance Mass Spectrometry. Anal. Chem. 75, 12671274 (2003) 